\title{
Incidência comparativa de câncer incidental de vesícula biliar em colecistectomias de urgência versus colecistectomias eletivas.
}

\section{Comparative incidence of incidental gallbladder cancer in emergency cholecystectomies versus in elective cholecystectomies.}

Welligton Ribeiro Figueiredo1; Rute Rocha Santos² ${ }^{2}$; Marlon Moreno da Rocha Caminha de Paula 3

\author{
R E S U M O
}

\begin{abstract}
Objetivo: analisar, comparativamente, a incidência de câncer incidental de vesícula biliar em colecistectomias de urgência versus colecistectomias eletivas realizadas em hospitais públicos de Teresina-PI. Métodos: estudo observacional descritivo, quantitativo, com delineamento transversal, cujo cenário foram dois hospitais públicos de Teresina-PI. Foram analisados 6.329 prontuários de pacientes submetidos à colecistectomia, entre janeiro de 2011 e dezembro de 2017. Os dados coletados foram divididos em dois grupos e confrontados estatisticamente através do teste Z para diferença entre proporções. Resultados: detectou-se câncer incidental da vesícula biliar em 6,53\% das colecistectomias de urgência e em 0,38\% das eletivas. Quanto ao sexo, observou-se que, na cirurgia de urgência, $69 \%$ eram mulheres e $31 \%$, homens, enquanto no procedimento eletivo, $78 \%$ eram mulheres e $22 \%$, homens. Quanto à idade, a maioria dos pacientes possuía mais de 60 anos e, entre estes, 69,3\% submetidos à colecistectomias de urgência e 82,6\%, à eletivas. O tipo histopatológico "adenocarcinoma" foi encontrado em 84,6\% das cirurgias de urgência e $100 \%$ das eletivas. Conclusão: câncer incidental de vesícula biliar foi mais frequente em colecistectomias de urgência em comparação às eletivas. O perfil dos pacientes com essa doença maligna nos dois tipos de procedimento foi do sexo feminino, maiores de 60 anos de idade e com diagnóstico histopatológico de adenocarcinoma.
\end{abstract}

Descritores: Colecistectomia. Neoplasias da Vesícula Biliar. Vesícula Biliar.

\section{INTRODUÇÃO}

O câncer de vesícula biliar é um tumor raro, com altas taxas de mortalidade. É o câncer de via biliar com menor sobrevida mediana no momento do diagnóstico, o mais comum do trato biliar e o quinto de maior incidência quando se elenca os cânceres gastrointestinais ${ }^{1,2}$.

Os pacientes são, na grande maioria, assintomáticos ou apresentam sintomas inespecíficos, como náuseas, dor abdominal ou perda de peso, que são indícios similares aos apresentados por pacientes com enfermidade benigna da vesícula biliar, o que dificulta o diagnóstico precoce. Apenas 30\% dos carcinomas de vesícula biliar são suspeitados no préoperatório, enquanto os demais são descobertos incidentalmente durante ou após a colecistectomia ${ }^{3}$.
A incidência do câncer de vesícula biliar incidental tem aumentado significativamente e atualmente é a apresentação mais frequente dessa entidade, com diagnóstico estabelecido pelo exame histopatológico da peça cirúrgica ${ }^{4}$.

Entre os fatores relacionados com a etiopatogênese do câncer da vesícula biliar, está a litíase, principalmente em coexistência com lama biliar, presente em $96 \%$ dos pacientes e sendo o principal fator de risco ${ }^{5}$. Obesidade, inflamação crônica, sexo feminino, idade maior do que 65 anos, etnia asiática ou afroamericana e valores elevados de fosfatase alcalina no pré-operatório também são relacionados a uma maior incidência de câncer de vesícula biliar .

Apesar do incremento no diagnóstico desses carcinomas incidentais, a maioria é diagnosticada em estágios avançados ${ }^{4}$. Por isso,

1 - Universidade Federal do Ceará, Faculdade de Medicina, Departamento de Cirurgia, Programa de Pós-Graduação em Cirurgia, Fortaleza, CE, Brasil. 2 - Faculdade Integral Diferencial/Facid-Devry, Curso de Medicina, Teresina, PI, Brasil. 3 - Universidade Federal do Piauí, Hospital Universitário (HU-UFPI), Serviço de Cirurgia Geral, Teresina, Pl, Brasil. 
quando, no momento da exploração cirúrgica, a doença é considerada irressecável, o que ocorre com na maioria dos casos, os procedimentos paliativos devem ser considerados ${ }^{7}$. Assim, os cirurgiões devem estar preparados para lidar com o câncer de vesícula biliar incidental, oferecendo o manejo mais conveniente de acordo com os achados. Além disso, torna-se relevante salientar que os diagnósticos tardios, particularmente relacionados ao estudo histopatológico inadequado ou à sua inobservância no período pós-operatório, se associam às baixas taxas de sobrevida, e isso tem destaque no que se refere à elaboração de medidas relacionadas à educação médica e à melhor assistência desses pacientes.

Este estudo procura analisar comparativamente a incidência de câncer incidental de vesícula biliar em colecistectomias de urgência versus colecistectomias eletivas realizadas em hospitais públicos de Teresina-PI, bem como, evidenciar a faixa etária e o sexo mais acometidos, e descrever o tipo histopatológico mais comum.

\section{MÉTODOS}

Trata-se de estudo de caráter observacional descritivo, quantitativo, com delineamento transversal para analisar, através do levantamento de dados de prontuários, os aspectos relativos ao câncer incidental de vesícula biliar em colecistectomias de urgência e eletivas. Foi desenvolvido de acordo com a Resolução 466, de 2012, do Conselho Nacional de Saúde (CNS), que define as diretrizes e normas regulamentadoras de pesquisas, envolvendo principalmente seres humanos. O projeto só foi iniciado após a apreciação e liberação do CEP FACID (Comitê de Ética em Pesquisa da Instituição), parecer CAAE no 88134318.0.0000.5211, e do CEP dos Hospitais relacionados à pesquisa, e mediante a assinatura do Termo de Compromisso de Uso de
Dados - TCUD pelos responsáveis das Instituições em que foi realizada a pesquisa.

O trabalho foi desenvolvido em dois hospitais públicos de referência de Teresina-PI, visto que são importantes centros da área cirúrgica e de pesquisa, no período de janeiro de 2011 a dezembro de 2017. Utilizou-se como critérios de inclusão os prontuários de todos os pacientes que foram submetidos à colecistectomia de urgência ou eletiva no período do estudo. Os critérios de exclusão foram: prontuários de pacientes com diagnóstico prévio de câncer de vesícula biliar, prontuários de pacientes que foram submetidos a outras cirurgias e retiraram ocasionalmente a vesícula biliar ou ainda prontuários preenchidos de maneira inadequada ou com dados insuficientes.

A coleta de dados foi realizada utilizando-se uma ficha de coleta de dados abrangendo as variáveis: faixa etária, sexo, CID-10 e resultado anatomopatológico, e os pacientes foram divididos em dois grupos: grupo A, que abrangeu os pacientes submetidos à colecistectomia eletiva, e que correspondeu aos dados coletados no hospital 1, e grupo B, que abrangeu os pacientes submetidos à colecistectomias de urgência, realizadas no hospital 2 .

Quanto à análise estatística, os dados foram organizados em planilhas do programa Microsoft Office Excel 2010 e apresentados na forma de gráficos e tabelas. Além disso, os dados correspondentes a cada técnica foram confrontados, comparando-se as variáveis analisadas, a fim de demonstrar diferenças estatisticamente significativas nos resultados entre cirurgias eletivas e de urgência. Para isso utilizou-se o teste Z para diferença entre proporções, por meio do software estatístico R Statistics, versão 3.4.4, com intervalo de confiança de 95\% e significância estatística de $\mathrm{p}<0,05$. 


\section{RESULTADOS}

Durante o período do estudo, 7.112 pacientes foram submetidos à colecistectomias eletivas e de urgência, dos quais 6.329 foram incluídos na análise. Destes, 5.931 foram submetidos à colecistectomia eletiva e 398 à cirurgia de urgência. Entre estes últimos, 26 (6,53\%) apresentaram diagnóstico pós-operatório de câncer de vesícula biliar através do exame anatomopatológico (Tabela 1).

Quanto aos achados anatomopatológicos referentes aos 5.931 pacientes que foram submetidos à colecistectomia eletiva, observa-se que $23(0,38 \%)$ apresentaram câncer de vesícula biliar (Tabela 2).
Ao comparar os dados coletados através do teste $Z$ para proporções, observou-se que existe uma diferença significativa entre a incidência dos cânceres de vesícula biliar diagnosticados após colecistectomia de urgência versus colecistectomia eletiva $(p<0,05)$, sendo esta enfermidade mais comum após cirurgia de urgência.

No que diz respeito ao sexo, dos 26 pacientes que tiveram diagnóstico de câncer incidental de vesícula biliar após colecistectomia de urgência, 18 eram do sexo feminino (69\%) e oito do sexo masculino $(31 \%)(p=0,0055)$. Já, em relação aos 23 pacientes submetidos à colecistectomia eletiva com diagnóstico de câncer incidental de vesícula biliar, 18 (78\%) eram do sexo feminino e cinco $(22 \%)$, do sexo masculino $(p=0,00013)$.

Tabela 1. Distribuição de doenças da vesícula biliar após colecistectomias de urgência.

\begin{tabular}{lcc}
\hline \multicolumn{1}{c}{ Achados anatomopatológicos } & \multicolumn{2}{c}{ Total } \\
\cline { 2 - 3 } & $\mathrm{N}$ & $\%$ \\
\hline Câncer de vesícula biliar & 26 & 6,53 \\
Vesícula em porcelana & 2 & 0,52 \\
Colecistite crônica em órgão escleroatrófico & 3 & 0,8 \\
Pólipo mucoso & 5 & 1,25 \\
Colecistite crônica com áreas de fibrose e calcificação & 6 & 1,5 \\
Colecistite crônica & 95 & 23,8 \\
Colecistite crônica com áreas de agudização & 125 & 31,4 \\
Colecistite aguda & 136 & 34,2 \\
Total & 398 & 100 \\
\hline
\end{tabular}

Tabela 2. Distribuição de doenças da vesícula biliar após colecistectomias eletivas.

\begin{tabular}{lcc}
\hline \multicolumn{1}{c}{ Achados anatomopatológicos } & \multicolumn{2}{c}{ Total } \\
\cline { 2 - 3 } & $\mathrm{N}$ & $\%$ \\
\hline Câncer de vesícula biliar & 23 & 0,38 \\
Vesícula em porcelana & 5 & 0,05 \\
Colecistite crônica em órgão escleroatrófico & 8 & 0,1 \\
Colecistite aguda & 38 & 0,2 \\
Colecistite crônica com áreas de fibrose e calcificação & 43 & 0,55 \\
Pólipo mucoso & 102 & 0,72 \\
Colecistite crônica com áreas de agudização & 5.710 & 1,8 \\
Colecistite crônica & 5.931 & 96,2 \\
Total & & 100 \\
\hline
\end{tabular}


Quando se convergem os dados do sexo mais acometido em colecistectomias de urgência versus colecistectomias eletivas, é possível concluir que, apesar de as amostras apresentarem valores diferentes, não houve diferença estatisticamente significativa $(p=0,4749)$, de modo que não se pode afirmar se o sexo feminino é mais acometido na cirurgia de urgência ou eletiva (Figura 1).

Para a análise da faixa etária, foram divididos grupos com critérios de corte: 0 a 30 anos, 31 a 60 anos, 61 a 70 anos, 71 a 80 anos e maiores de 80 anos. Dos 26 pacientes submetidos à colecistectomia de urgência que tiveram diagnóstico anatomopatológico de câncer de vesícula biliar, um (3,8\%) estava na faixa de
0 a 30 anos, sete $(26,9 \%)$ na de 31 a 60 anos, oito $(30,8 \%)$ possuíam entre 61 e 70 anos, quatro $(15,4 \%)$ estavam na faixa de 71 a 80 anos e seis $(23,1 \%)$ eram maiores de 80 anos.

Quanto aos 23 pacientes diagnosticados com câncer incidental de vesícula biliar pós-cirurgia eletiva, quatro $(17,4 \%)$ possuíam entre 31 e 60 anos, 11 (47,8\%) tinham entre 61 e 70 anos, cinco $(21,7 \%)$ pertenciam à faixa etária entre 71 e 80 anos e três $(13,1 \%)$ eram maiores de 80 anos.

Ao comparar as idades de pacientes submetidos à colecistectomias de urgência e a colecistectomias eletivas, evidenciou-se que não havia diferença estatisticamente significativa entre eles ( $p=0,2771$ ) (Figura 2).

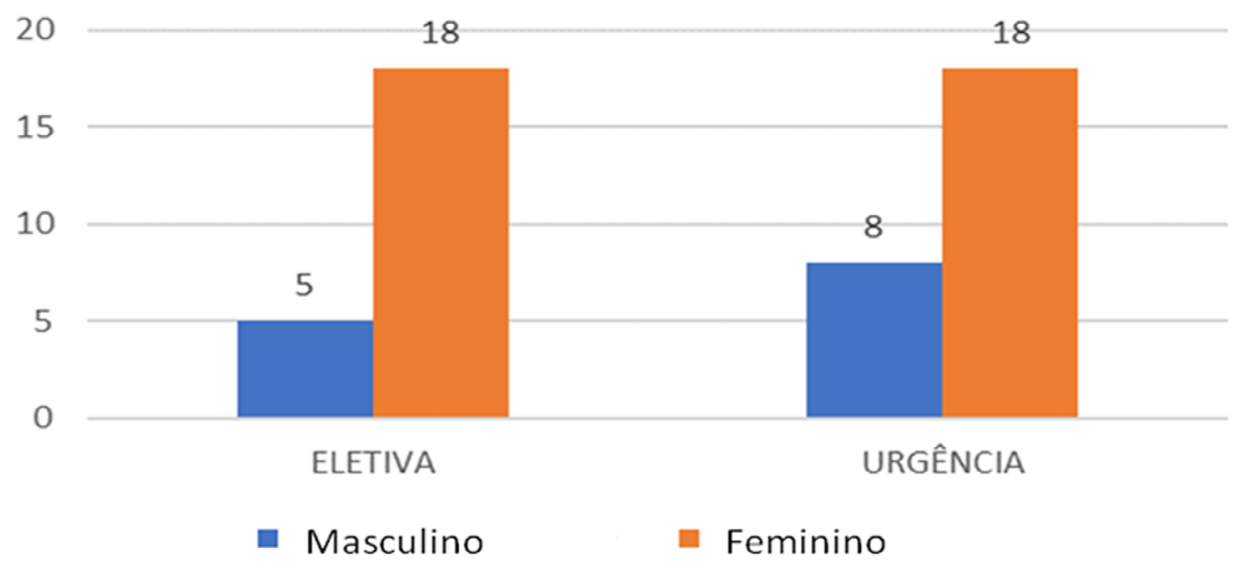

Figura 1. Distribuição por sexo de pacientes com câncer incidental de vesícula biliar.

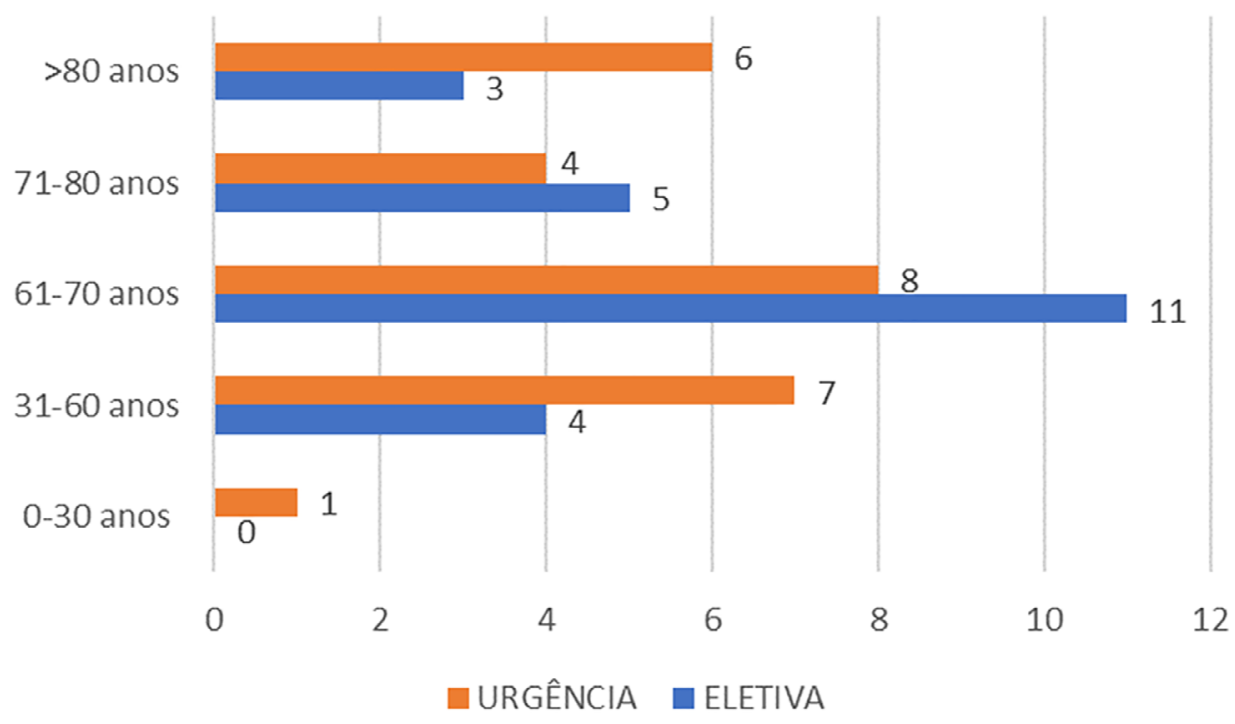

Figura 2. Distribuição por idade de pacientes com câncer incidental de vesícula biliar. 
Em relação ao tipo histopatológico, dos 26 cânceres incidentais de vesícula biliar póscolecistectomia de urgência, quatro $(15,4 \%)$ eram carcinomas epidermoides e 22 (84,6\%) eram adenocarcinomas, sendo um (3,85\%) metastático, $16(61,5 \%)$ pouco diferenciados, quatro $(15,4 \%)$ moderadamente diferenciados e um $(3,85 \%)$ bem diferenciado. Ao comparar os tipos histopatológicos identificados no diagnóstico de pacientes com câncer pós colecistectomias de urgência observou-se que o mais comum foi o adenocarcinoma pouco diferenciado $(p=0,00063)$.

Já, dos 23 casos de câncer de vesícula biliar pós-colecistectomia eletiva, todos tinham adenocarcinomas, sendo um $(4,35 \%)$ do tipo mucinoso, dois $(8,7 \%)$ papilíferos, três (13\%) pouco diferenciados, dez $(43,5 \%)$ moderadamente diferenciados e sete $(30,45 \%)$ bem diferenciados. Relacionando os tipos histopatológicos, observou-se que, apesar de haver diferença amostral entre o adenocarcinoma moderadamente diferenciado e o adenocarcinoma bem diferenciado, que foram os dois mais encontrados, ambos foram considerados igualmente os mais prevalentes ( $p=0,3595)$. Já, ao comparar esses dois tipos histopatológicos com os demais, houve uma diferença estatística significativa $(p=0,0012)$, ratificando que eles foram os mais comuns.
O tipo histopatológico mais comum, tanto nos cânceres diagnosticados pós-colecistectomias de urgência $(p<0,05)$ quanto naqueles evidenciados pós-cirurgia eletiva $(p<0,05)$, foi o adenocarcinoma, e, ao se convergir os dados mais prevalentes da colecistectomia de urgência versus a eletiva, pôde-se afirmar que houve uma diferença estatisticamente significativa $(p=0,049)$, de modo que a cirurgia eletiva teve uma incidência maior de adenocarcinoma quando comparada à cirurgia de urgência (Figura 3).

\section{DISCUSSÃO}

O câncer de vesícula biliar, apesar de raro, é responsável por $80 \%$ a $95 \%$ dos casos de afecções malignas envolvendo o trato biliar, sendo o câncer mais comum nessa região ${ }^{8}$. Apesar disso, poucos estudos têm sido relatados sobre aspectos relacionados ao câncer de vesícula biliar, pelo fato de ainda ser relativamente raro em países do ocidente ${ }^{9}$. Nesse contexto, o exame histopatológico dos espécimes da vesícula biliar é um passo importante para a confirmação do diagnóstico' .

Os dados referentes aos diagnósticos de câncer pós-colecistectomia de urgência vão de encontro à maior parte da literatura, como por exemplo, o estudo de Noriega Usi et al. ${ }^{10}$,

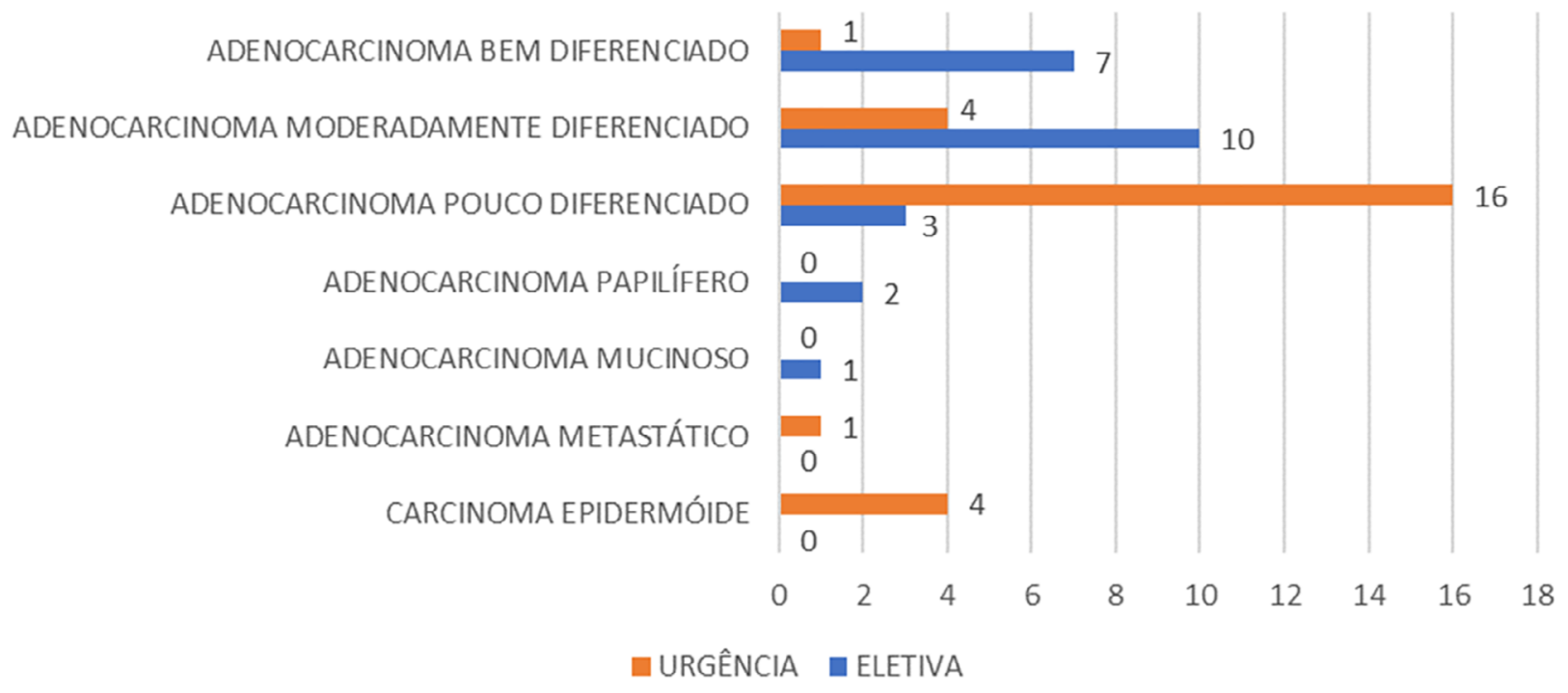

Figura 3. Distribuição por tipo histopatológico de câncer incidental de vesícula biliar. 
que avaliou 903 colecistectomias realizadas ao longo de cinco anos e encontrou uma incidência de câncer incidental de vesícula biliar de 0,4\%. Já, as taxas relacionadas à cirurgia eletiva corroboram com os dados encontrados na maior parte da literatura mundial, como por exemplo, o estudo de MeirellesCosta et al. ${ }^{11}$, que demonstrou que a ocorrência do câncer incidental de vesícula biliar pode variar de $0,3 \%$ a $2 \%$ de todas as colecistectomias realizadas por condições benignas.

A evidência de que essa enfermidade é mais comum após colecistectomia de urgência também foi demonstrada em outros estudos, como o de Torres et al. ${ }^{12}$, que sugeriu que o estresse crônico, prolongado, da vesícula biliar, que ocorre em pacientes sintomáticos e que são submetidos à cirurgia de urgência, aumenta o tempo de exposição à oncogenes e aumentam o grau de displasia epitelial.

Em relação ao sexo mais acometido, é possível observar de maneira estatisticamente significativa que o sexo feminino tem maior incidência em ambos os procedimentos cirúrgicos, como demonstrado no estudo de Ishak et al. ${ }^{13}$, no qual o câncer de vesícula biliar apresentou, entre as mulheres, uma taxa de 79\%. Dincel et al. ${ }^{1}$, em sua pesquisa, evidenciaram que o sexo feminino tem uma frequência quatro vezes maior de câncer de vesícula biliar quando comparado ao sexo masculino. O estudo de Salazar et al. ${ }^{14}$ também demonstrou que há maior incidência e maior coeficiente de mortalidade no sexo feminino.

O risco aumentado de câncer de vesícula biliar em mulheres é explicado pela maior incidência de colelitíase em mulheres quando comparado com homens. Além disso, a exposição mais prolongada a hormônios sexuais femininos também pode ser um fator predisponente. Portanto, a idade mais jovem na menarca, primeira gravidez precoce, gravidezes múltiplas e um período prolongado de reprodução, por serem situações de exposição prolongada aos agentes hormonais, também podem aumentar o risco de câncer do trato biliar ${ }^{11}$.
Ademais, as mulheres buscam atendimento com mais frequência do que os homens e isso pode justificar a maior incidência no sexo feminino.

Quanto à faixa etária, a maior parte da literatura vai ao encontro do que é evidenciado aqui, como no estudo de Tian et al. ${ }^{15}$, que concluiu que a média de idade dos pacientes com essa patologia era de 61 anos, e na pesquisa de PaisCosta et al. ${ }^{9}$, que evidenciou que a média de idade dos pacientes acometidos com câncer incidental de vesícula biliar foi de 71 anos.

O estudo de Meirelles-Costa et al. ${ }^{11}$ também demonstrou que a incidência de lesões précancerosas, bem como, de câncer, deve aumentar com a idade, por conta do maior tempo de exposição à mutações e produção de oncogenes, sendo a maior incidência entre os pacientes com mais de 60 anos, confirmando o que é demonstrado nesta pesquisa. O envelhecimento está associado ainda a um maior tempo de exposição a fatores de risco, como infecções, sequência adenoma-carcinoma, obesidade, ativação de fatores genéticos, os quais aumentam a probabilidade de diagnóstico incidental de câncer.

Por fim, esse estudo demonstra que o tipo histopatológico mais comum é o adenocarcinoma, havendo concordância com o que é evidenciado na maior parte da literatura, como nos estudos de Kalita et al. ${ }^{16}$, que mostraram que, dos 18 cânceres incidentais de vesícula biliar encontrados, 15 eram adenocarcinomas, e na pesquisa de ApodacaRueda et al. ${ }^{17}$, que revelou adenocarcinoma em 90\% dos casos de câncer incidental de vesícula biliar.

Concluímos, assim, que o câncer incidental de vesícula biliar tem maior incidência na cirurgia de urgência quando comparada à eletiva, e que, em ambas as modalidades cirúrgicas analisadas, a neoplasia é mais frequente no sexo feminino, em pacientes acima de 60 anos de idade, e o tipo histopatológico mais comum o adenocarcinoma. 
O achado ocasional do câncer de vesícula biliar é mais comum do que se imagina, e isso reforça a importância do retorno ambulatorial com revisão do exame histopatológico da vesícula biliar, pois a ausência dessa medida se associa ao diagnóstico de câncer biliar em estágios avançados e baixas taxas de sobrevida.

\title{
A B S T R A C T
}

\begin{abstract}
Objective: to comparatively analyse the incidence of incidental gallbladder cancer in emergency cholecystectomies versus in elective cholecystectomies performed in public hospitals in Teresina city, Piaui state (PI). Methods: descriptive, quantitative, observational, cross-sectional study, whose scenarios were two public hospitals in Teresina-Pl. We analysed 6,329 medical records of patients undergoing cholecystectomy between January 2011 and December 2017. The collected data were divided into two groups and statistically compared using Z-test for difference between proportions. Results: incidental gallbladder cancer was detected in $6.53 \%$ of emergency cholecystectomies and in $0.38 \%$ of elective ones. Regarding gender, it was observed that in emergency surgeries $69 \%$ of patients were women and $31 \%$ men, while in elective procedures 78\% were women and 22\% men. Regarding age, most patients were over 60 years old, and, among these, 69.3\% underwent emergency cholecystectomies and $82.6 \%$ underwent elective cholecystectomies. The histopathological type "adenocarcinoma" was found in $84.6 \%$ of patients who underwent emergency surgeries and in $100 \%$ of patients who underwent elective surgeries. Conclusion: incidental gallbladder cancer was more frequent in urgent cholecystectomies compared to elective cholecystectomies. The profile of patients with this malignant disease in both types of procedure was female, older than 60 years, and with histopathological diagnosis of adenocarcinoma.
\end{abstract}

Keywords: Cholecystectomy. Gallbladder Neoplasms. Gallbladder.

\section{REFERÊNCIAS}

1. Dincel O, Goksu M, Hatipoglu HS. Importance of routine histopathological examination of a gallbladder surgical specimen: unexpected gallbladder cancer. J Can Res Ther. 2018;14(6):1325-9.

2. Siegel $R$, Desantis $C$, Jemal A. Colorectal cancer statistics, 2014. CA Cancer J Clin. 2014;64(2):104-17.

3. Goetze T, Paolucci V. Does laparoscopy worsen the prognosis for incidental gallbladder cancer? Surg Endosc. 2006;20(2):286-93.

4. Ledndoire J, Gil L. Carcinoma de la vesícula biliar. Enciclopedia Cirugía Digestiva. F. Galindo y colab. Sociedad Argentina de Cirugía Digestiva. 2015; tomo IV-463:1-22.

5. Cariati A, Piromalli E, Cetta F. Gallbladder cancers: associated conditions, histological types, prognosis, and prevention. Eur J Gastroenterol Hepatol. 2014;26(5):562-9.

6. Solaini L, Sharma A, Watt J, losifidou S, Chin Aleong JA, Kocher HM. Predictive factors for incidental gallbladder dysplasia and carcinoma. J Surg Res. 2014;189(1):17-21.

7. Shukla PJ, Barreto SG. Gallbladder cancer: we need to do better! Ann Surg Oncol. 2009;16(8):2084-5.
8. Mazer LM, Losada HF, Chaudhry RM, VelazquezRamirez GA, Donohue JH, Kooby DA, et al. Tumor characteristics and survival analysis of incidental versus suspected gallbladder carcinoma. J Gastrointest Surg. 2012;16(7):1311-7.

9. Pais-Costa SR, Farah JFM, Artigiani-Neto R, Franco MIF, Martins SJ, Goldenberg A. Adenocarcinoma da vesícula biliar: avaliação dos fatores prognósticos em 100 casos ressecados no Brasil. ABCD. Arq Bras Cir Dig. 2012;25(1):13-9.

10. Noriega Usi VM, Álvarez Sánchez M, Hernández Méndez R, Rodríguez Luna R, Bieletto Trejo EO, Fenig Rodriguez J. Carcinoma de vesícula biliar incidental después de colecistectomía. Análisis de los hallazgos patológicos a cinco años. Acta Méd. Grupo Ángeles. 2016;14(4):196-200.

11. Meirelles-Costa ALA, Bresciani CJC, Perez RO, Bresciani BH, Siqueira SAC, Cecconello I. Are histological alterations observed in the gallbladder precancerous lesions? Clinics (São Paulo). 2010;65(2):143-50.

12. Torres OJM, Barbosa ES, Pantoja PB, Diniz MCS, da Silva JRS, Czeczko NG. Prevalência ultra-sonográfica de litíase biliar em pacientes ambulatoriais. Rev Col Bras Cir. 2005;32(1):47-9. 
13. Ishak G, Ribeiro FS, Costa DS, Bahia LAC, Dias EM, Assumpção PP. Câncer de vesícula biliar: experiência de 10 anos em um hospital de referência da Amazônia. Rev Col Bras Cir. 2011;38(2):100-4.

14. Salazar M, Ituarte C, Abriata MG, Santoro F, Arroyo G. Gallbladder cancer in South America: epidemiology and prevention. Chin Clin Oncol. 2019;8(4):32.

15. Tian $Y H$, Ji $X$, Liu $B$, Yang GY, Meng $X F$, Xia HT, et al. Surgical treatment of incidental gallbladder cancer discovered during or following laparoscopic cholecystectomy. World J Surg. 2015;39(3):746-52.

16. Kalita $D$, Pant $L$, Singh $S$, Jain $G$, Kudesia $M$, Gupta K, et al. Impact of routine histopathological examination of gall bladder specimens on early detection of malignancy - a study of 4,115 cholecystectomy specimens. Asian Pac J Cancer Prev. 2013;14(5):3315-8.
17. Apodaca-Rueda M, Cazzo E, De-Carvalho RB, Chaim EA. Prevalence of gallbladder cancer in patients submitted to cholecystectomy: experience of the University Hospital, Faculty of Medical Sciences, State University of Campinas - UNICAMP. Rev Col Bras Cir. 2017;44(3):252-6.

Recebido em: 25/09/2019

Aceito para publicação em: 29/10/2019

Conflito de interesse: nenhum.

Fonte de financiamento: nenhuma.

\section{Endereço para correspondência:}

Rute Rocha Santos

E-mail: ruters_@hotmail.com ruterocha1014@gmail.com

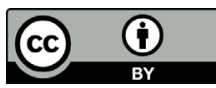

